\title{
Efecto de la responsabilidad social corporativa en la reputación de las organizaciones: una revisión sistemática
}

\author{
Anggy Daniela Castaño Ramírez \\ Samuel Arias Sánchez ${ }^{* *}$
}

Fecha de recibido: 31 de marzo de 2020 Fecha de aprobado: $1 .^{\circ}$ de septiembre de 2020

Para citar este artículo: Castaño Ramírez, A. D., \& Arias Sánchez, S. (2021). Efecto de la responsabilidad social corporativa en la reputación de las organizaciones: Una revisión sistemática. Revista Universidad \& Empresa, 23(40), 1-25. https://doi.org/10.12804/

revistas.urosario.edu.co/empresa/a.8859

\section{Resumen}

La responsabilidad social corporativa se entiende como la forma de actuar responsable de una organización para con sus stakeholders o grupos de interés. Esta variable se ha hecho objeto de investigación por generar valor en las empresas a través de los activos intangibles, como la reputación corporativa, que hace referencia a la percepción de los grupos sobre las compañías. A pesar de la amplia actividad investigativa, no se encontró revisión alguna que hubiera analizado sistemáticamente su relación en las diferentes publicaciones. Por este motivo, se hizo necesario el desarrollo de la presente investigación, para estudiar el efecto que podría tener la responsabilidad social corporativa en la reputación. Por medio de

* Licenciada en Psicología y máster en Gestión de Recursos Humanos por la Universidad de Sevilla (España). En la actualidad, trabaja en el Departamento de Recursos Humanos de una empresa multinacional y colabora con el Laboratorio de Actividad Humana del Departamento de Psicología Experimental de la Facultad de Psicología de la Universidad de Sevilla. Correo electrónico: anggydanielac@gmail.com

** Doctor en Psicología. Máster en Psicología del Trabajo y de las Organizaciones. Máster en Cerebro y Conducta por la Universidad de Sevilla (España). En la actualidad, forma parte del Departamento de Psicología Experimental, de la Facultad de Psicología de la Universidad de Sevilla, como profesor del área de Metodología de las Ciencias del Comportamiento. Correo electrónico: samuel@us.es 
Efecto de la responsabilidad social corporativa en la reputación de las organizaciones: una revisión sistemática

una metodología cuantitativa se analizaron diecinueve artículos empíricos, a efectos de demostrar una relación directa y positiva entre ambas variables, en la cual se han detectado que intervienen otras variables mediadoras como transparencia, edad, confianza en la marca, entornos externos, entre otras.

Palabras clave: responsabilidad social corporativa; reputación; intangibles; revisión sistemática; variables mediadoras.

\title{
Effect of Corporate Social Responsibility on the Reputation of Organizations: A Systematic Review
}

\begin{abstract}
Corporate Social Responsibility is understood as the way to act responsibly on the part of an organization towards its stakeholders or interest groups. This variable has been investigated for generating value in companies through intangible assets, such as the Corporate reputation that refers to the perception of groups about companies. Despite the extensive research activity, no review was found that systematically analyzed their relationship in the different publications. For this reason, the development of this investigation became necessary to study the effect that corporate social responsibility could have on reputation. Through a quantitative methodology, nineteen empirical articles were analyzed, demonstrating a direct and positive relationship between both variables in which other mediating variables such as transparency, age, brand confidence, and external environments, among others, have been detected.
\end{abstract}

Keywords: Corporate social responsibility; reputation, intangibles; systematic review; mediating variables.

\section{Efeito da responsabilidade social corporativa na reputação das organizações: uma revisão sistemática}

\section{Resumo}

Responsabilidade social corporativa se entende como a forma responsável de atuação de uma organização para com seus stakeholders ou grupos de interesse. Esta variável tem sido objeto de investigação por gerar valor nas empresas através de ativos intangíveis, como a reputação corporativa, que faz referência à percepção dos grupos sobre a companhia. Apesar da ampla pesquisa realizada, não se encontrou nenhuma revisão que tenha analisado sistematicamente sua relação nas diferentes publicações acessadas. Por este motivo, se fez necessário o desenvolvimento da presente pesquisa, para estudar o efeito que a responsabilidade social corporativa possa ter sobre a reputação. Através de uma metodologia quantitativa se analisaram 19 artigos empíricos, demonstrando uma relação direta e positiva entre ambas as variáveis, na qual também se detectaram a intervenção de outras variáveis mediadoras, como a transparência, idade, confiança na marca, entorno externo, entre outras.

Palavras-chave: responsabilidade social corporativa; reputação; intangíveis; revisão sistemática; varáveis mediadoras. 


\section{Introducción}

La aplicación de prácticas de responsabilidad social corporativa (RSC) se ha convertido en la actualidad en una necesidad para las organizaciones. Es ya un elemento clave para su gestión, ya que por medio de estas se crea y se mantiene una buena relación con todos sus interlocutores, debido a que son afectadas por las decisiones corporativas. Dicha relación ayuda a alcanzar un óptimo desarrollo económico en la compañía y genera, en un principio, la tendencia a investigar el efecto de la Rsc en el desempeño económico, dejando a un lado el efecto que se generaba en los intangibles de la organización, como es el caso de la reputación corporativa (RC) (Vázquez, 2012).

En los últimos años se han presentado distintas formas de aproximarse a este constructo tan escurridizo (García-Santos \& Madero-Gómez, 2016). Autores como Caroll (1979, p. 500) exponen la Rsc como "la responsabilidad social de los negocios que abarca las expectativas económicas, legales, éticas y discrecionales que la sociedad tiene de las organizaciones en cierto periodo de tiempo". Wood (1991, p. 695), por otra parte, argumenta que "la idea básica de la responsabilidad social corporativa es que los negocios y la sociedad están entretejidos, en vez de ser entidades distintas". Para Hopkins (2003), en la Rsc se destaca la importancia mediar de una manera ética con los diferentes stakebolders de una empresa, lo que García-Santos \& Madero-Gómez (2016) extrapolan al trato y a una responsabilidad compartida que característico de una sociedad civilizada. Otra definición importante de este concepto es la de "obligaciones de los hombres de negocios de perseguir aquellas políticas, tomar aquellas decisiones o seguir aquellas líneas de acción, las cuales son deseables en términos de objetivos y valores de nuestra sociedad" (Bowen, 2013, p. 6).

Debido a la diversidad de conceptualizaciones encontradas, en su revisión Dahlsrud (2008) estableció que generalmente se identifican cinco dimensiones en común en las principales definiciones de Rsc, correspondientes a la ambiental, la social, la económica, la de los stakeholders y la voluntariedad. La ambiental hace hincapié en la naturaleza o aspectos relacionados, como cuidar y obtener un medio ambiente más limpio o considerar las preocupaciones ambientales en las operaciones de negocios. La dimensión social establece una relación entre empresa y sociedad, donde se integran las preocupaciones sociales en sus operaciones comerciales, considerando el alcance completo de su impacto 
en la sociedad, a la vez que se contribuye a que esta sea cada vez mejor. La dimensión económica se centra en los aspectos socioeconómicos o financieros, incluyendo la Rsc en términos de una operación empresarial, con el fin de favorecer el desarrollo económico y preservar la rentabilidad de la empresa que la aplique. En la dimensión de stakebolders se reconocen y se hacen valer los intereses de los distintos grupos con los que se relaciona la organización. Finalmente, en la voluntariedad se hace referencia a todas aquellas acciones de Rsc que no están prescritas por la ley basadas en valores éticos.

En definitiva, la Rsc es una de las áreas de estudio con mayor importancia en la actualidad, no solo por el hecho de ser una tendencia empresarial del presente siglo, debido a que genera sostenibilidad a través de una economía sustentable, sino también porque cada vez aparecen nuevos procesos, mecanismos e ideologías que permiten aplicar de forma diferente esta disciplina (Pérez et al., 2016). En esta misma línea, según Henríquez Larrarte y Oreste Burgos (2015), el aumento de la importancia y preservación de las prácticas responsables y el desarrollo académico de estas se evidencia en su reconocimiento a través de proyectos o términos como normas iso, huella verde, memoria de sostenibilidad, entre otros, que suelen ser utilizados en todo el mundo.

Aunque normalmente se asocie la responsabilidad social únicamente con empresas de gran tamaño o multinacionales, Torassa (2010) expone que estos principios también son aplicables a cualquier empresa, debido a la naturaleza de la actividad. Se puede considerar que la RSC se ha convertido en un factor de supervivencia empresarial y aquellos que lo descuiden ponen en riesgo tanto su legitimidad social como su papel económico. Esta es una cuestión que intentará determinar el presente estudio. De esta forma, se entiende que las políticas de RSC afectan positivamente el rendimiento de las organizaciones, pero de la misma forma han despertado la preocupación por fortalecer la reputación corporativa. En el contexto español se ha demostrado que nueve de cada diez personas tienden a pagar productos que destinan un porcentaje de sus ingresos a proyectos sociales y siete de cada diez a aquellos que invierten en lo ecológico. Así es como se evidencia que es necesario estudiar no solo la Rsc, sino también la Rc, además de su efecto conjunto (Valenzuela et al., 2015).

A partir de la publicación del ranking sobre las empresas más admiradas "Most Admired Companies" de la revista norteamericana Fortune, en 1982, se empieza a dar valor en las 
empresas a la Rc (González, 2017). Según Alloza et al. (2013), a partir de ese momento, el concepto de RC adquiere más peso y evoluciona desde una perspectiva de imagen hasta una perspectiva evaluativa. Se la ha ido prestando cada vez mayor interés a este concepto, que se podría definir como "el conjunto de evaluaciones colectivas, suscitadas por el comportamiento corporativo, en las distintas audiencias, que motivan sus conductas de apoyo u oposición" (Alloza et al., 2013, p. 96).

Según Curras-Pérez (2010), se ha evidenciado que en la actualidad empresarial la RC siempre se ve vinculada a acciones positivas como mejoría de ventas, buen rendimiento financiero, éxito corporativo, atracción y retención de talento, diferenciación frente a la competencia y muchos más beneficios, que hacen importante la gestión integral de la empresa para alcanzar la reputación deseada. Uno de los casos en los cuales se ha evidenciado la importancia que se le ha brindado a la RC en los últimos años es en el ranking de empresas con mejor reputación en España (Monitor Empresarial de la Reputación Corporativa, 2019), pues son las compañías con más éxito en el mercado las que lideran este ranking.

Considerando la importancia y las implicaciones de la RC, es de interés saber qué factores influyen en alcanzar lo que se considera una buena reputación. Carrió (2013) describió cuatro factores clave: la calidad (de los productos, la gestión, los empleados, las relaciones, etc.), el rendimiento (económico, financiero, de innovación, etc.), la RSC (social, medioambiental, fiscal, legal, etc.) y el atractivo (autenticidad, diferenciación, relevancia, confianza, etc.).

En esta misma línea, pero desde el punto de vista de la Rsc, se ha demostrado que esta se convierte en un elemento positivo con beneficios reales tangibles e intangibles; de ahí la gran cantidad de estudios en distintas partes del mundo que se interesan por comprobar la correlación positiva entre los resultados económicos y financieros y la Rsc. Esto ha generado en las empresas el deseo de obtener un mayor desarrollo económico y de reputación a través de estas prácticas. Por tal motivo, es primordial abarcar, desde otra perspectiva, los beneficios de las acciones socialmente responsables, como en el caso de los intangibles, como la imagen y reputación que también generan valor para las organizaciones. Estas logran afectar el comportamiento del consumidor hacia una actitud positiva, pues a través de ellas se obtiene una importante ventaja competitiva para las empresas, superando por ejemplo al producto, venta o servicio, que puede ser imitado por la competencia (Molina et al., 2017). En un estudio en 
un gran banco español se describió una relación circular entre RSC y RC, y se ha constituido un departamento de Rcc con la premisa "un comportamiento responsable es clave para una buena reputación" (De Castro, 2008). No obstante, esta experiencia está contextualizada en un tipo de organización muy específica y no se podría hacer una extrapolación directa a otros casos, por lo que consideramos que se hace interesante explorar la literatura de estudios empíricos que hayan vinculado la Rsc de una organización con su RC.

En esta línea, algunos autores se han interesado por estudiar la relación entre RSC y RC. Sin embargo, en el caso de Aksak et al. (2016), difirieron en su metodología y objetivo propuesto, ya que con una revisión literaria buscaban obtener un análisis conceptual y no empírico. Estos autores identificaron tres teorías principales para explicar esta relación: la primera es la de los stakeholders, donde las razones de compra de los consumidores se basan no solo en lo económico, sino también en lo social, como aquellos esfuerzos de RSC de una empresa que influyen en la comunidad, pues los clientes no solo son sensibles a los precios, sin que también tienen preocupaciones sociales y ambientales que influyen en sus decisiones de compra. La segunda es la de atribución, que explica las atribuciones y, por ende, la RC desde un proceso cognitivo donde las personas asignan una explicación a un evento observado; incluso puede ser negativo, donde las personas piensen que la RSC se utiliza solo para obtener ganancias financieras, o positivo, donde se ven estas actividades como beneficiosas para la empresa y grupos interesados. Por último, la teoría de valor expone que las acciones de RSC deben estar basadas en los valores centrales de la empresa, siendo esencial determinar las prioridades de valor detrás de las actividades de RSC, pues así mismo podría determinarse la RC.

Aunque se pueden encontrar algunas teorías que intentan explicar la relación entre RSC y RC, son pocas las investigaciones que estudian si es una relación directa o indirecta, determinada por una u otras variables. Algunos autores sugieren que los stakebolders reaccionan positiva o negativamente a las actividades de RSC y, por ende, a la reputación de empresa, cuestionándose los motivos detrás de esas actividades. Este planteamiento puede explicar la intervención de otros factores para generar esta relación, exponiendo que, para alcanzar la Rc responsable, las partes interesadas deben percibir que la empresa se ha comportado adecuadamente y con buenas intenciones. Es decir, las comunicaciones de las actividades de RSc han sido transparentes y han limitado los efectos sociales 
negativos, de forma confiable y preocupándose genuinamente por la sociedad en general (Park et al., 2014).

Del mismo modo, se evidencia, según el cone (2013), que las nuevas generaciones tienden a crear, e incluso a recomendar, con una reputación más positiva aquellas marcas que han realizado acciones de apoyo social y medioambiental, lo que conlleva posteriormente no solo una intención de compra, sino un deseo de incorporación a esas empresas, retención del talento y, por ende, un alto rendimiento financiero de las empresas. Eso se puede deber a que las generaciones previas disponen de más información de las organizaciones con las que se relacionan, al estar más conscientes del poder que tienen sus decisiones y los beneficios y consecuencias derivadas de estas (Peñalosa Otero \& López Celis, 2018).

Tal como hemos visto, es innegable la estrecha relación entre RSC y RC, su importancia y relevancia para cualquier organización laboral, así como su influencia en el rendimiento y manteamiento a largo plazo de las y los empleados. Empero, no se ha encontrado una revisión de las diferentes investigaciones al respecto que permitan conocer en profundidad y confirmar la magnitud de la posible relación RSC-RC, así como el papel de otras variables que influyan. Por ello, el objetivo propuesto en este trabajo ha sido el de llevar a cabo una revisión sistemática de las publicaciones empíricas que hayan vinculado la RSC y la RC, al igual que analizar la forma en que se han planteado dichas investigaciones, los resultados presentados, así como sus conclusiones.

\section{Método}

Antes de iniciar la búsqueda bibliográfica, se desarrolló la pregunta de investigación siguiendo el formato picos, junto a la lista de términos tesauro para facilitar y especificar dicha búsqueda, determinando como palabras clave en inglés: organizaciones, empresas, RSC, RC y estudio empírico, utilizando los operadores booleanos and y or. Una vez formulado el problema y realizada la búsqueda a través de la base de datos Web of Science, escogida por ser una de las principales bases de datos académicas con publicaciones científicas de cualquier disciplina de conocimiento, se seleccionaron artículos siguiendo el protocolo 
expuesto en el artículo de Perestelo-Pérez (2013) "Standards on How to Develop and Report Systematic Reviews in Psychology and Health”.

Para ser incluidos en esta revisión, los estudios debían cumplir con los siguientes criterios de inclusión establecidos: a) estar en idioma español o inglés, b) haber sido publicados en la franja temporal 2015-2019 y c) ser un estudio empírico.

En primera instancia, se obtuvieron un total de 134 artículos, entre los cuales a partir de la lectura de títulos y resúmenes se excluyeron los referidos a temas no relacionados, igualmente aquellos que abarcaran las variables (RSC y RC) pero de forma individual sin indagar el efecto de una sobre otra. También se tuvo en cuenta como criterio de exclusión los estudios con metodología de tipo cualitativo y teórico, obteniendo como muestra final un total de 19 artículos que cumplen con los criterios establecidos. Detallamos el número de artículos por fase en el proceso de cribado en la figura 1.

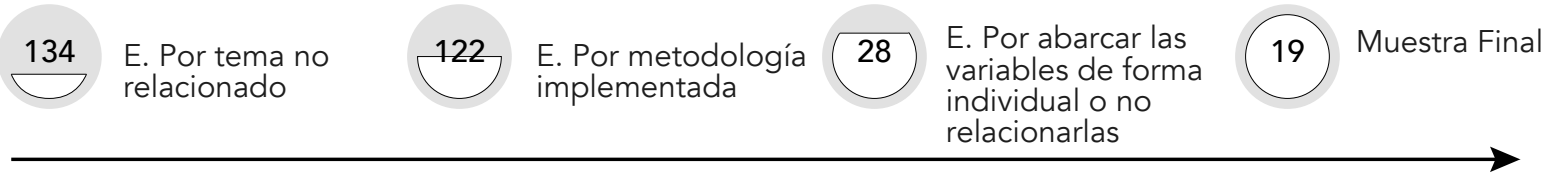

Figura 1. Número de artículos por fase en el proceso de cribado

Fuente: Elaboración propia

Luego de tener identificados los estudios primarios (véase tabla 1), se realizó una lectura crítica de estos para proceder a la extracción de datos. Se diseñó una plantilla para el registro de datos descriptivos: nombre del artículo, autores, muestra, número de citas, país y año. Cabe resaltar que para conocer el número de citas se utilizó Google Académico (con corte el 2 de julio de 2019), que era el único dato no encontrado en el artículo.

Posteriormente, con otra plantilla se extrajeron datos referentes a los resultados; estos correspondían al valor beta de la relación entre RSC y RC. Para este proceso, inicialmente, fue necesario distinguir en cada publicación cuál de los tres posibles métodos se utilizó para estudiar la relación, ya que podía ser correlación, regresión múltiple o el del modelo de ecuaciones estructural. Finalmente, se extrajeron los datos de aquellos artículos que consideraban variables mediadoras entre RSC y RC, siendo necesario identificar la variable y el valor beta correspondiente a la mediación, o en unos de los casos el valor de correlación. 


\section{Resultados}

Al observar la figura 1 y la tabla 1 se identifica que una vez realizada la búsqueda bibliográfica y la filtración acorde con los criterios de inclusión y exclusión, se obtuvieron como muestra final 19 artículos, con los cuales se establece el escenario para los resultados de la presente revisión sistemática. Para iniciar es necesario destacar que la totalidad de los artículos escogidos analizaron sus datos por medio de correlación, regresión múltiple o modelo de ecuaciones estructurales, a efectos de investigar la relación entre RSC y RC, debido a que con estas técnicas se analizan las asociaciones entre diversos constructos, que proporcionan evidencia de los efectos directos e indirectos positivos y significativos de las variables en estudio.

Tabla 1. Datos descriptivos de los estudios primarios

\begin{tabular}{|c|c|c|c|c|c|c|}
\hline Cód. & Autores & Artículo & $\mathbf{N}$ & País & Año & Cita \\
\hline A1 & Lee et al. & $\begin{array}{l}\text { An investigation of the effects of corporate social responsibility } \\
\text { on corporate reputation and customer loyalty - evidence from } \\
\text { the Taiwan non-life insurance industry }\end{array}$ & 362 & Taiwán & 2017 & 12 \\
\hline A2 & Su et al. & $\begin{array}{l}\text { Corporate social responsibility: Findings from the Chinese hospi- } \\
\text { tality industry }\end{array}$ & 451 & China & 2017 & 22 \\
\hline A3 & $\begin{array}{l}\text { Arslanagic } \\
\text { y Zabkar }\end{array}$ & $\begin{array}{l}\text { Hold me responsible. The role of corporate social responsibility } \\
\text { and corporate reputation for client-perceived value }\end{array}$ & 228 & Europa & 2017 & 8 \\
\hline A4 & Choongo & $\begin{array}{l}\text { A Longitudinal Study of the Impact of Corporate Social } \\
\text { Responsibility on Firm Performance in SMEs in Zambia }\end{array}$ & 153 & Zambia & 2017 & 20 \\
\hline A5 & $\begin{array}{l}\text { Jalilvand } \\
\text { et al. }\end{array}$ & $\begin{array}{l}\text { Social responsibility influence on customer trust in hotels: medi- } \\
\text { ating effects of reputation and word-of-mouth }\end{array}$ & 300 & Irán & 2017 & 18 \\
\hline A6 & $\begin{array}{l}\text { Aguilera } \\
\text { Caracuel y } \\
\text { Guerrero } \\
\text { Villegas }\end{array}$ & $\begin{array}{l}\text { How Corporate Social Responsibility Helps MNEs to Improve } \\
\text { their Reputation. The Moderating Effects of Geographical } \\
\text { Diversification and Operating in Developing Regions }\end{array}$ & 102 & EE. UU. & 2018 & 11 \\
\hline A7 & $\begin{array}{l}\text { González } \\
\text { et al. }\end{array}$ & $\begin{array}{l}\text { The effect of technological posture and corporate social respon- } \\
\text { sibility on financial performance through corporate reputation }\end{array}$ & 76 & España & 2018 & - \\
\hline A8 & $\begin{array}{l}\text { Vlastelica } \\
\text { et al. }\end{array}$ & $\begin{array}{l}\text { How Corporate Social Responsibility Affects Corporate } \\
\text { Reputation: Evidence from an Emerging Market }\end{array}$ & 688 & Serbia & 2018 & 2 \\
\hline A9 & $\begin{array}{l}\text { Fernández } \\
\text { Sánchez } \\
\text { et al. }\end{array}$ & $\begin{array}{l}\text { The relationship between corporate social responsibility and cor- } \\
\text { porate reputation in a turbulent environment: Spanish evidence } \\
\text { of the lbex35 firms }\end{array}$ & 26 & España & 2015 & 16 \\
\hline A10 & $\begin{array}{l}\text { Taghian } \\
\text { et al. }\end{array}$ & $\begin{array}{l}\text { A stakeholder approach to corporate social responsibility, repu- } \\
\text { tation and business performance }\end{array}$ & 196 & Australia & 2015 & 53 \\
\hline A11 & Kim et al. & $\begin{array}{l}\text { Corporate Brand Trust as a Mediator in the Relationship be- } \\
\text { tween Consumer Perception of CSR, Corporate Hypocrisy, and } \\
\text { Corporate Reputation }\end{array}$ & 520 & Seúl & 2015 & 46 \\
\hline
\end{tabular}




\begin{tabular}{|c|c|c|c|c|c|c|}
\hline Cód. & Autores & Artículo & $\mathbf{N}$ & País & Año & Cita \\
\hline A12 & $\begin{array}{l}\text { Fatma et } \\
\text { al. }\end{array}$ & $\begin{array}{l}\text { Building company reputation and brand equity through CSR: the } \\
\text { mediating role of trust }\end{array}$ & 303 & India & 2015 & 63 \\
\hline A13 & $\begin{array}{l}\text { Vidaver y } \\
\text { Brønn }\end{array}$ & $\begin{array}{l}\text { Reputation, Responsibility, and Stakeholder Support in } \\
\text { Scandinavian Firms: A Comparative Analysis }\end{array}$ & 581 & $\begin{array}{l}\text { Países } \\
\text { escandi- } \\
\text { navos }\end{array}$ & 2015 & 69 \\
\hline A14 & $\begin{array}{l}\text { Reverte } \\
\text { et al. }\end{array}$ & $\begin{array}{l}\text { The influence of corporate social responsibility practices on orga- } \\
\text { nizational performance: evidence from Eco-Responsible Spanish } \\
\text { firms }\end{array}$ & 133 & España & 2016 & 55 \\
\hline A15 & Lin et al. & $\begin{array}{l}\text { How Does Environmental Irresponsibility Impair Corporate } \\
\text { Reputation? A Multi-Method Investigation }\end{array}$ & 57 & China & 2016 & 32 \\
\hline A16 & $\begin{array}{l}\text { Del Brío y } \\
\text { Lizarzaburu } \\
\text { Bolaños }\end{array}$ & $\begin{array}{l}\text { CSR Actions in Companies and Perception of Their Reputation by } \\
\text { Managers: Analysis in the Rural Area of an Emerging Country in } \\
\text { the Banking Sector }\end{array}$ & 112 & Perú & 2018 & 1 \\
\hline A17 & $\begin{array}{l}\text { Baraibar- } \\
\text { Díez y } \\
\text { Sotorrío }\end{array}$ & $\begin{array}{l}\text { The mediating effect of transparency in the relationship between } \\
\text { corporate social responsibility and corporate reputation }\end{array}$ & 22 & España & 2018 & 9 \\
\hline A18 & $\begin{array}{l}\text { Engizek y } \\
\text { Yasin }\end{array}$ & $\begin{array}{l}\text { How CSR and overall service quality lead to affective commitment: } \\
\text { mediating role of company reputation }\end{array}$ & 522 & Turquía & 2017 & 10 \\
\hline A19 & $\begin{array}{l}\text { Vercic y } \\
\text { Ćorić }\end{array}$ & $\begin{array}{l}\text { The relationship between reputation, employer branding and } \\
\text { corporate social responsibility }\end{array}$ & 550 & Croacia & 2018 & 6 \\
\hline
\end{tabular}

Fuente: elaboración propia.

Al analizar el país de cada uno de los estudios escogidos, se evidenció, en primer lugar, que el $47 \%$ de estos corresponde al territorio europeo, y el $32 \%$, al continente asiático; por ende, son significativos los resultados en cuanto a esta área. Asimismo, se distinguió que algunos de los artículos más citados se encuentran inmersos en estos porcentajes. Esto evidencia que dichos estudios tienen un alto valor de repercusión en otros trabajos académicos; por el contrario, los estudios con menos citación pertenecen al 10\%, que a su vez son los correspondientes al continente americano, aunque se resalta que el poco impacto evidenciado se podría deber a su reciente fecha de publicación.

Es necesario destacar que catorce de los estudios primarios utilizaron en sus muestras stakebolders internos o externos para evaluar sus variables, detallando más adelante cada una de ellas, excepto el artículo A8, que tuvo en cuenta ambas muestras. Los cinco estudios restantes (correspondientes al A6, A9, A13, A15 y A17) implementaron análisis de datos empíricos tomando como referencia para sus muestras información de bases de datos que miden la RSC y RC, como KLD, Fortune, Monitor Empresarial de la Reputación Corporativa, Observatorio de la Rsc, Reputation Institute, Mercado de Valores de China, Csmar, Ministerio de Protección 
Ambiental de China, Thomson Reuters, entre otros indicadores, que tienen en cuenta la utilización de cada uno acorde con el espacio geográfico donde se desarrolló la investigación.

\subsection{Relación directa entre responsabilidad social corporativa y reputación corporativa}

Como se mencionó, las investigaciones escogidas difirieron en el método implementado; por esta razón, se dividieron los 19 estudios en dos grupos para poder analizar sus datos. En primer lugar, en la tabla 2 se representan las relaciones informadas en 7 de los 19 artículos, entre la reputación y los distintos tipos de RSC consideradas. Los valores extraídos en este grupo corresponden a análisis de correlación o regresión múltiple. Respecto a aquellos que evaluaron la RSC por dimensiones, en la primera línea de la tabla 2 se evidencia la correspondiente a cada caso, y a partir de la tercera columna de izquierda a derecha, la responsabilidad económica, legal, ética, discrecional, ambiental y social. Las dos columnas restantes corresponden a la consistencia e intensidad con la que se ha aplicado la RSC.

Tabla 2. Resultados de correlación y análisis de regresión múltiple

\begin{tabular}{|c|c|c|c|c|c|c|c|c|c|c|c|}
\hline & Cód. & RSC & $\begin{array}{c}\text { RSC } \\
\text { Antes }\end{array}$ & $\begin{array}{c}\mathrm{R} . \\
\text { econ. }\end{array}$ & R. legal & R. ética & $\begin{array}{c}\text { R. } \\
\text { discr. }\end{array}$ & R. amb. & $\begin{array}{c}\text { R. } \\
\text { Social }\end{array}$ & $\begin{array}{c}\text { Cons. } \\
\text { RSC }\end{array}$ & $\begin{array}{l}\text { Int. } \\
\text { RSC }\end{array}$ \\
\hline \multirow{8}{*}{ 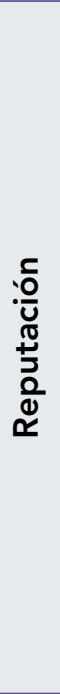 } & A1 & & & $0.204^{* * *}$ & $0.253^{* x+x}$ & $0.369^{*+* t+x}$ & 0.010 & & & & \\
\hline & A6 & $0.38^{\star \star \star}$ & & & & & & & & & \\
\hline & $\begin{array}{l}\text { A8 } \\
\text { Ciu. }\end{array}$ & & & & $0.512^{* * *}$ & & & $0.408^{* \star *}$ & $0.403^{* * *}$ & & \\
\hline & $\begin{array}{l}\text { A8 } \\
\text { Stak. }\end{array}$ & & & & $0.466^{* *}$ & & & $0.436^{* * *}$ & $0.303^{* * x}$ & & \\
\hline & A9 & & & & & & & & & $-0.163^{*}$ & $0.581^{*}$ \\
\hline & A13 & $\begin{array}{l}\text { Nor: } 0.63 \\
\text { Din: } 0.90 \\
\text { Sue: }_{* \star x} 0.78\end{array}$ & & & & & & & & & \\
\hline & $\begin{array}{l}\text { A15 } \\
\text { Loss } \\
\text { RC } \\
\end{array}$ & $-0.07^{*}$ & $-0.03^{*}$ & & & & & & & & \\
\hline & A19 & 0.224 & & & & & & & & & \\
\hline
\end{tabular}

${ }^{\star} p<0.1 ;{ }^{* \star} p<0.05 ;{ }^{* \star \star} p<0.01 ;{ }^{* \star \star \star} p<0.001$.

Nor: Noruega; Din: Dinamarca; Sue: Suecia; Cons: Consistencia; Int: Intensidad.

Fuente: elaboración propia. 
Se puede observar que aquellos estudios en los cuales se evaluó la Rsc de forma global (A6, A13, A15 y A19) respecto a su influencia en la RC mostraron una relación positiva y estadísticamente significativa, excepto el estudio A15, que evidenció una correlación negativa en dos tiempos: con un valor correspondiente a un lapso anterior de $\beta=-0.03$ y otro correspondiente a la actualidad de la investigación de $\beta=-0.07$. Sin embargo, se resalta que dichos valores se obtuvieron de evaluar los índices de Rsc con la pérdida de reputación, siendo estos datos inversos al resto de estudios que evaluaban los efectos positivos en RC, lo que concluiría, así como los otros estudios, que la rsc contribuye a aumentar y mejorar la reputación.

Asimismo, se destaca en estos artículos que su muestra se basó en empresas multisectoriales y en índices de bases datos, excepto en esto último el A19. Ello indica que sin importar el sector o actividad en la que esté inmersa la empresa, la relación entre RSC y RC va a ser positiva y significativa, pues son datos tomados de una muestra conformada por diversas empresas que no son aplicables a un único sector. Otros datos aportados no presentes en la tabla 2 y que conviene mencionar, debido a los valores de correlación más altos, son los correspondientes al A13, que tras haber establecido una relación significativa en el estudio, decidió examinar los indicadores de RSC y su aportación en porcentaje a la RC en comparación con otras variables, y así obtuvo una media del 39.5\% para Noruega, del $41 \%$ para Dinamarca y del $40.6 \%$ para Suecia, siendo por ende la variable que más aporte brinda a la reputación, luego la calidad del producto y servicio, con un porcentaje global del $23.9 \%$.

Contrario a los artículos mencionados, que realizaron sus estudios por medio de un análisis de bases datos con empresas multisectoriales, los artículos A1 y A8 utilizaron muestras especificas correspondientes a los grupos de interés de la organización analizada. Por un lado, en el A1, con los clientes de empresas de seguros en Taiwán, y en el A8, con ciudadanos consumidores de empresas locales en Serbia y grupos específicos como la comunidad empresarial, medios de comunicación, administración pública y sociedad civil. Ello nos brinda un análisis restrictivo aplicable solo en estas poblaciones, debido a la especificación de sus características.

Continuando con estos dos estudios, la RSC se evaluó desde varias dimensiones que coincidían en la responsabilidad legal. Respecto a esta, en ambos se demostró una relación 
positiva y significativa con la Rc. Esa fue especial en el A8, ya que es la dimensión que mayor efecto tiene sobre la reputación y es particularmente fuerte con un valor $\beta=0.512$, desde la perspectiva de los ciudadanos, y un valor $\beta=0.466$ desde los grupos específicos de stakeholders. En este mismo artículo y en ambos grupos (ciudadanos y stakebolders), el resto de las dimensiones (correspondiente a la ambiental y social) tienden a tener una correlación moderada a fuerte, pero igualmente positiva y significativa. Esta misma correlación se evidencia en el A1 con la dimensión económica y ética. Sin embargo, respecto a la discrecional, que corresponde a aquellas actividades empresariales que no son obligatorias ni requeridas por la ley, los resultados con un valor $\beta=0.010$ indican que no hay un efecto significativo en la RC.

Para finalizar este primer grupo de resultados está el estudio A9. En este se evaluó la RSC por sus características de aplicación, correspondientes a la consistencia e intensidad. La primera se entiende como el periodo en el que se aplica o se ha mantenido la Rsc, y la segunda, como el volumen de actividad responsable llevada a caba por las empresas. Esta última es la que se correlaciona positivamente con la RC, sin ser significativa la consistencia.

El segundo grupo de datos reflejado en la tabla 3 corresponde a los artículos que implementaron para su análisis el modelo de ecuaciones estructurales. Al igual que el grupo anterior, dos artículos tuvieron en cuenta evaluar la Rsc desde algunas de sus posibles dimensiones. En este caso, la dimensión analizada con la Rc en ambos artículos (A4 y A16) fue la ambiental. Según los valores obtenidos, se evidencia una relación positiva en ambos estudios, aunque solo significativa en el A4, lo que valida parcialmente el efecto que pueden generar las acciones ambientales en la reputación. Asimismo, se evaluaron dimensiones como la social en el A4, en la que no se indicó una compatibilidad significativa, y la salud en el A16, donde se demostró la influencia de aplicar acciones de RSc orientadas al área de la salud sobre la RC, pues fue estadísticamente significativo con un valor de $\beta=0.517$.

Hay que destacar que estos estudios pudieron estar influidos por la muestra utilizada, debido a que en el sector pyme, en el caso del A4, según Choongo (2017), la RC puede tener menos impacto que en las grandes empresas, y en el A16, al abarcar el área rural, la teoría ya afirma que la salud es unos de los factores más críticos para las personas que viven en esta área, a causa de su deficiencia en desarrollo, siendo evidente que la salud sea más influyente que las acciones ambientales (Del Brío y Lizarzaburo Bolaños, 2018). 
Tabla 3. Resultados de modelos de ecuaciones estructurales

\begin{tabular}{|c|c|c|c|c|c|}
\hline & Cód. & RSC & Social & Ambiental & Salud \\
\hline \multirow{12}{*}{ 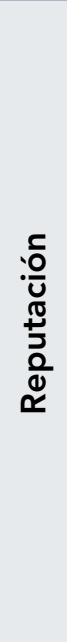 } & A2 & $0.63^{\star \star \star}$ & & & \\
\hline & A3 & $0.60^{\star \star \star}$ & & & \\
\hline & A4 & & $0.149 p>0.10$ & $0.269^{\star}$ & \\
\hline & A5 & $0.454^{\star \star \star}$ & & & \\
\hline & A7 & $0.540 * \star \star *$ & & & \\
\hline & A10 & $0.53^{* *}$ & & & \\
\hline & A11 & 0.31 ** & & & \\
\hline & A12 & $0.48^{*}$ & & & \\
\hline & A14 & $0.774^{\star \star}$ & & & \\
\hline & A16 & & & 0.106 & $0.517^{\star}$ \\
\hline & A17 & -0.081 & & & \\
\hline & A18 & $0.453^{\star \star}$ & & & \\
\hline
\end{tabular}

${ }^{*} p<0.05 ;{ }^{* *} p<0.01 ;{ }^{* *} p<0.001$.

Fuente: elaboración propia.

Una vez analizados los datos del artículo A4 y A16 de la tabla 3, se continuó con los 10 artículos restantes. Estos difieren de los anteriores, debido a que evaluaron de forma global la variable RSC. Las muestras utilizadas en este caso fueron específicas correspondiente a sus respectivos grupos interesados, a excepción del A17, que fue el único estudio de este grupo que desarrolló su investigación a partir de la base de datos de Thomson Reuters y el Monitor Empresarial de la Reputación Corporativa. Igualmente, se observa que los sectores en los que se realizaron las investigaciones fueron: hotelería (A2 y A5), servicios profesionales (A3), bancario (A12, A16 y A18), ambiental (A4 y A7) y multisectorial (A10, A11, A14 y A17).

En el camino de evaluar la relación directa que va desde la RsC a la RC, los resultados en este segundo grupo coincidieron en nueve de los diez artículos, esto es, demostraron en todos un efecto significativamente directo y positivo en la relación de estas variables, luego de obtenerse una relación que tiende a ser de moderada a fuerte, pues sus valores oscilaron entre $\beta=0.774$ y $\beta=0.31$. Finalmente, el único valor contrario a la mayoría de los datos expuestos fue el $\beta=-0.081$, correspondiente al estudio A17. Este valor contradice la relación positiva hasta ahora probada, por lo que es preciso destacar que en este estudio se tuvo en cuenta la adición de otra variable con la posibilidad de que fuera la mediadora entre Rsc y $\mathrm{RC}$, probando tras el efecto directo no sustancial que hay una mediación completa de la cual se hablará en el segmento posterior. 


\subsection{Variables mediadoras entre responsabilidad social y reputación}

Para dar por cumplido el objetivo de esta revisión sistemática, también se analizaron aquellos estudios en los que se proponía un modelo de relación indirecta entre RSC y RC, pues cuestionaban la posibilidad de que dicha relación estuviera mediada por otras variables. Se encontraron un total de nueve estudios de los 19 con este planteamiento, los cuales obtuvieron el valor de la mediación a través de los procesos de regresión y modelo de ecuaciones estructurales. Al igual que los datos anteriores, se extrajo el valor beta (tabla 4) correspondiente a cada variable mediadora, a excepción del estudio A9, en el cual se extrajo el dato de $\mathrm{R}^{2}$, el cual explica el valor porcentual y predictivo del modelo propuesto en ese caso.

Tabla 4. Resultados de la influencia de las variables mediadoras de la relación RSC-RC

\begin{tabular}{|c|c|c|c|c|c|c|c|c|c|}
\hline Variable & A1 & $\mathrm{A} 2$ & A6 & A8 & A9 & A11 & A12 & A14 & A17 \\
\hline $\begin{array}{l}\text { Imagen de } \\
\text { marca }\end{array}$ & $0.271^{\star \star \star}$ & & & & & & & & \\
\hline Ingresos & & $\begin{array}{l}\text { Bajo }=0.39 \\
\text { Alto }=0.60 \\
\star\end{array}$ & & & & & & & \\
\hline Educación & & - & & & & & & & \\
\hline $\begin{array}{l}\text { Diversificación } \\
\text { geográfica de } \\
\text { la empresa }\end{array}$ & & & 0.06 & & & & & & \\
\hline $\begin{array}{l}\text { Operar en } \\
\text { regiones en } \\
\text { desarrollo }\end{array}$ & & & $0.26^{\star \star \star}$ & & & & & & \\
\hline Edad & & & & $\begin{array}{l}\text { Legal } \\
-30=0.65^{\star \star} \\
\text { Ambiental } \\
-30=0.43^{\star \star} \\
60+=0.49^{\star \star} \\
\underline{\text { Social }} \\
46-60=0.48^{\star \star}\end{array}$ & & & & & \\
\hline $\begin{array}{l}\text { Entorno } \\
\text { económico } \\
\text { externo }\end{array}$ & & & & & $R^{2}=0.92$ & & & & \\
\hline $\begin{array}{l}\text { Confianza } \\
\text { del cliente } \\
\text { en la marca }\end{array}$ & & & & & & $0.23^{*}$ & 0.19 & & \\
\hline
\end{tabular}




\begin{tabular}{|c|c|c|c|c|c|c|c|c|c|}
\hline Variable & $\mathrm{A} 1$ & $\mathrm{~A} 2$ & A6 & A8 & A9 & A11 & A12 & A14 & A17 \\
\hline Innovación & & & & & & & & $0.219 * \star$ & \\
\hline Transparencia & & & & & & & & & $0.198^{\star *}$ \\
\hline
\end{tabular}

En la tabla 4 se exponen las variables mediadoras que fueron consideradas en cada investigación. En primer lugar, se aprecia que en el estudio A6 se tuvieron en cuenta dos posibles variables: una de ellas fue la diversificación geográfica de la empresa, de la cual no se encontró evidencia de tener un efecto moderador significativo, y la otra analizó el hecho de que las empresas multinacionales operen en regiones en desarrollo (Asia Oriental y el Pacífico, África, Asia Central y América Latina), la que en efecto se asumió como una variable moderadora de la relación RSC-RC, que será positiva cuando se dé un alto nivel de operación en los países en desarrollo. Es decir, si las empresas deciden implementar sus proyectos de RSC en regiones que se encuentran en esa transición de crecimiento social, económico, gubernamental, etc., su impacto en la RC será mayor, contrario a su aplicación en otros territorios que difieren de estas características.

En cuanto a la confianza del cliente en la marca, fue la única variable que se mencionó en dos artículos (A11 y A12). En ambos, después de comprobar una relación directa RSC-RC, se estimó que hay una mediación parcial y estadísticamente significativa a través de la confianza. Asimismo, en el estudio A1 se tuvo en cuenta la imagen de la marca, pero sin la confianza de los casos anteriores. Los autores de este artículo consideraron que la percepción de los clientes de la imagen de marca estaba creada a partir de la RSC de su empresa y, además, tenía un efecto significativo en la reputación que otorgaban a la organización.

Otra de las variables que demostró mediar la relación fue la innovación, con un efecto indirecto de 0.219, que indicó ser significativo. Ante este resultado, en el estudio A14 mencionan que dicha mediación a través de esta variable se hace efectiva en empresas manufactureras o de fabricación, pues lograron comprobar que en empresas no correspondientes a este sector el efecto indirecto fue negativo, con un valor de -0.170 . 
En esta misma línea también se quiso indagar en el A17 la relación indirecta de la RSC -RC; pero esta vez a través de la transparencia. Baraibar-Díez y Sotorrío (2018), autores de esta investigación, se planteaban que las empresas deben comunicar sus acciones de RSC para alcanzar la RC, pero dicha comunicación debe hacerse de manera adecuada y confiable, lo cual supone hacerlo de forma transparente. Esta hipótesis fue comprobada al finalizar el estudio, pues sus resultados apoyaron una mediación completa con un efecto indirecto y significativo, contrario al efecto directo que no fue sustancial, como se expuso en el apartado anterior de resultados.

Los siguientes dos estudios por analizar comprobaron la mediación de sus variables a la vez que se realizó una comparación. En primer lugar, se demostró en el A2 que la educación, sin mencionar datos o valores al respecto, no influye en esta relación. Por el contrario, en este mismo se comprobó que los ingresos de los clientes de una empresa sí pueden moderar el efecto de la RSC en la RC. Esta mediación se hace notable entre aquellos consumidores que presenten ingresos más altos y, por ende, será más probable que los ricos asuman la RSC como un impulsor de reputación. Seguidamente, en el estudio A8 el factor considerado fue la edad de los ciudadanos, donde la relación RSC-RC va a tender a ser más fuerte en personas mayores de 30 años. Se tuvo en cuenta la RSC desde la dimensión legal y ambiental, aunque esta última también es relevante en la población mayor de 60 años. Por último, para hacer referencia a la dimensión social, los resultados demuestran que la relación tiene mayor efecto en personas con edades comprendidas entre 46 y 60 años.

Cabe mencionar que la última variable moderadora identificada en esta revisión fue el entorno económico externo (EEE) de la empresa. El efecto mediador de esta variable se valida a través del valor de $\mathrm{R}^{2}=0.92$, el cual es entendido en porcentaje. Hace referencia al efecto o fuerza del modelo en su conjunto ( $\mathrm{RSC} \rightarrow \mathrm{EEE} \rightarrow \mathrm{RC}$ ). Es decir, en un $92 \%$ este modelo establece que la RSC a través de la EEE predice el cambio en la RC. Se concluye así que, a pesar de presentarse una crisis financiera, no se afectará negativamente la relación RSC-RC, pues contrario a esto la intensidad y consistencia de la RSC tiende a aumentar significativamente $\mathrm{y}$, por ende, también el efecto en la reputación.

Para culminar los resultados es necesario destacar que considerando la relación entre RSC y RC, estas variables mediadoras nos brindan información y validan la existencia de un efecto indirecto en la mencionada relación. Se distingue la variable de la edad como 
aquella que mayor impacto mediador puede generar en la reputación tras aplicar acciones socialmente responsables.

\section{Discusión}

En esta investigación, el objetivo era llevar a cabo una revisión sistemática para estudiar el efecto de la RSC en la RC e identificar, a su vez, las posibles variables mediadoras existentes en esta relación, analizando el efecto directo e indirecto ellas. Respecto a la primera, los datos tuvieron una visión homogénea e indican efectivamente, como lo expuso Carrió (2013), que la Rsc es un factor clave para generar directamente Rc. A partir de esta idea, se brinda al mundo empresarial el punto de partida para añadir en las empresas ese valor diferenciador en el mercado, el cual ya no depende solo de un producto o servicio brindado, sino de la percepción que los stakebolders tienen sobre la forma de ser y actuar de la compañía.

Una vez comprobado el efecto positivo de la RSC en la Rc, se comprueba igualmente los beneficios que esta relación conlleva para el sector empresarial. Según Peñalosa Otero y López Celis (2018), no solo se limitan a lo económico, igualmente generan que nuevos y futuros profesionales deseen pertenecer a las compañías con buena reputación y se retenga el talento ya obtenido. Por esta razón, es significativo discutirlo, debido a la importancia que se le debe brindar al reconocimiento de todos los actores inmersos en los grupos de interés, en el momento de aplicar acciones de Rsc. Se debe entender que no se habla solo de clientes, medios de comunicación, gobierno o agentes externos, sino de todas aquellas partes que son afectadas por las acciones de las empresas, incluyendo a actores internos como lo son los empleados, accionistas, dueños, etc. Por ello, al identificar una relación positiva entre las dos variables y al ser estos grupos los que determinan la Rc, se considera necesario dirigir las acciones también a favor de los intereses de esta comunidad.

En este mismo sentido, se comprobó la teoría de los stakebolders expuesta en Aksak et al. (2016), según la cual se debe considerar de qué manera la Rsc va a influir en la sociedad, lo cual implica tener en cuenta las preocupaciones sociales y ambientales de esta. Ese fue 
el caso de dos estudios en los que se trabajó con los intereses de las sociedades donde están inmersas las organizaciones y en que se benefició su RC. Uno de los estudios fue el A6, que comprobó que el operar en regiones en desarrollo actúa como variable mediadora; se deduce que esto es posible debido a los territorios donde se aplicó (Asia Oriental y el Pacífico, África, Asia Central y América Latina), ya que corresponden a lugares con amplias necesidades como desigualdad, pobreza, contaminación, sobrepoblación, etc.; o en el caso del A16, el cual comprobó que la salud mediaba la relación al aplicarse en áreas rurales, pues son sectores críticos que carecen de avances en esta área.

Asimismo, se identificaron factores asociados a la relación indirecta como la confianza y la transparencia, las cuales pueden desempeñar un rol importante y significativo como mediadoras. Este resultado coincidió con lo expuesto por Park et al. (2014), quienes mencionan que las personas tienden a dudar de las intenciones detrás de las acciones responsables. Para crear una buena reputación se hace necesario que haya transparencia en sus implementaciones y que se garantice que han actuado de forma adecuada, creando a la vez confianza hacia la organización. A partir de este hecho, se considera que el avance tecnológico en la actualidad puede contribuir a crear esta confianza. Por medio de esa tecnología se va teniendo conocimiento de todas las acciones aplicadas, sus impactos y valoraciones. Así, también concuerda que la edad sea una variable mediadora, pues son las nuevas generaciones las que más acceden a estas herramientas digitales, que facilitan la transmisión de información a cualquier persona y lugar en el mundo.

Al considerar todos los datos analizados hasta el momento, podemos establecer que una relación directa es capaz de generar mayor impacto si las empresas deciden estudiar factores mediadores en la relación, sobre todo antes de aplicar la Rsc. Por lo tanto, es recomendable que las empresas se impliquen y conozcan las verdaderas necesidades y características de la comunidad donde se encuentran o, igualmente, identifiquen comunidades externas que necesiten de ayudas sociales, medioambientales $u$ otras acciones adheridas a la Rsc sobre las cuales puedan actuar. A partir de esta premisa las empresas contribuyen a disminuir necesidades y problemas reales, a fin de lograr un mayor impacto en los beneficios alcanzados y, por ende, en su reputación.

Los resultados de este análisis inducen a pensar que las empresas podrían ampliar sus grupos de interés si deciden aplicar la Rsc en comunidades externas que pueden favorecer 
la innovación con nuevos proyectos al identificar factores no tenidos en cuenta por otras compañías de la competencia, y que los grupos beneficiados por la Rsc podrían tener una percepción más positiva al hacerse conscientes de que las empresas actúan a favor de sus intereses y no se limitan a únicamente aumentar su cuenta de resultados. Para conseguir estos indudables beneficios intangibles, es necesario que las organizaciones actúen desinteresadamente y hagan de la RSC un espacio de retribución y agradecimiento hacia sus grupos de interés y sociedad como tal, por contribuir al crecimiento de sus empresas. Se deben concienciar respecto a que mediante estos colectivos, que utilizan y adquieren sus servicios, han logrado mantenerse en el mercado.

A pesar de estos resultados, entre las limitaciones encontradas se resalta que la teoría aportaba la existencia de varias dimensiones de la Rsc, como ambiental, social, económica, stakeholders y voluntariedad (Dahlsrud, 2008), que contaron con poca presencia en esta revisión sistemática. Solo cuatro estudios tuvieron en cuenta algunas de ellas. Se hace necesario en futuras investigaciones que se indague sobre el efecto individual de cada una en la reputación, identificando si esta variable tiene mayor impacto, dependiendo de la implementación de una u otra o confirmando la dependencia de la aplicación global de la RSC sobre la obtención de una buena reputación.

Asimismo, al ser la reputación una variable multidimensional, como lo expuso Carrió (2013), es preciso en un futuro investigar en qué medida influye cada uno de los factores (rendimiento, responsabilidad, atractivo y calidad) en la creación de esta variable y no solo desde la rsc, como fue el caso. Este punto de partida para nuevas investigaciones es necesario al mencionar que cada una, a pesar de ser independientes, están relacionadas entre sí, y en esa interacción surge la Rc. Finalmente, como limitación de la presente revisión se destaca la generalización en la muestra escogida, ya que se tuvo información de diversos continentes e igualmente diversos sectores. Por tal razón, se dificulta brindar de forma específica cómo puede gestionarse la RSC desde una actividad de negocio exacta para obtener la reputación deseada. Sin embargo, como lo expuso Torassa (2010), la Rsc es un "factor de supervivencia empresarial”, por lo cual sin importar el sector debería aplicarse en todas las empresas del siglo XXI.

Así, a partir de este estudio es posible brindar un área de trabajo desde los recursos humanos, pues actualmente se debe potenciar, concienciando a las empresas de que a 
través de este departamento se logrará el desarrollo óptimo de estas políticas, al promover la colaboración de todo el capital humano debido a que son acciones que atañen a todos, y este departamento es el encargado de comunicarlas e involucrar a cada uno de los trabajadores en ellas de la mano de nuevas tecnologías, creando y fomentando la cultura responsable de la empresa desde una gestión interna con empleados comprometidos con los objetivos de la organización, que posteriormente se transmitirá de forma externa mejorando la percepción que tiene la sociedad de las entidades y el valor reputacional.

\section{Conclusiones}

El objetivo de la presente revisión se dio por cumplido, al determinar un efecto positivo entre las variables RSC y RC. Se comprobó y aportó desde este estudio que la relación puede estar determinada por el tipo de acción responsable que se aplica en beneficio de los stakeholders. Si las empresas desean aumentar su RC a partir de la Rsc, deben tener en cuenta los intereses de la sociedad donde está inmersa, evitando aplicarla sin un análisis previo de los intereses de su comunidad interna y externa, ya que ambas determinarán su $\mathrm{RC}$, según los beneficios que hayan recibido de la RSC. El hecho de estudiar esos intereses también permitirá la creación de nuevos proyectos innovadores que no solo beneficie su reputación, sino que además la posicionaría mejor entre la competencia del mercado.

A pesar de comprobar la relación directa entre las variables, se evidenció que puede ser determinante la intervención de variables mediadoras para alcanzar un mayor impacto y beneficio a largo plazo. Se recomienda involucrar las tecnologías de información en la comunicación de las acciones aplicadas, porque con ello habrá un mayor alcance de información a la comunidad y divulgación. De igual forma, las comunicaciones deben caracterizarse por la transparencia, componentes éticos y claridad de la información, con la cual construirá una confianza del cliente en la marca, que podrá trasmitirse entre y a otros distintos grupos de interés.

Finalmente, y respecto a la limitación encontrada sobre la generalización de la muestra, la cual no permite identificar qué sector empresarial se puede beneficiar en mayor 
o menor medida del efecto de la responsabilidad social en la reputación, se determinó que sin importar la actividad que desempeñen las empresas siempre van a obtener un beneficio en su reputación y, en consecuencia, serán compañías socialmente responsables. Debido a que la RC determina cómo se percibe una organización, desempeñará un papel significativo, aunque no total, en la determinación del éxito o fracaso de las empresas, siendo uno de los generadores de valor más importantes en la actualidad. A partir de este estudio es preciso decir que la RC puede actuar como un factor extrínseco que motive a las empresas a aplicar lo que conocemos como Rsc.

Los autores confirman no tener conflictos de intereses en esta publicación, y que la presente investigación no ha recibido subvención alguna de ningún organismo de financiación de los sectores público, comercial o no lucrativo.

\section{Referencias}

Aguilera-Caracuel, J., \& Guerrero-Villegas, J. (2018). How corporate social responsibility helps MNES to improve their reputation: The moderating effects of geographical diversification and operating in developing regions. Corporate Social Responsibility and Environmental Management, 25(4), 355-372. https://doi.org/10.1002/csr.1465

Aksak, E., Ferguson, A., \& Duman, A. (2016). Corporate social responsibility and csR fit as predictors of corporate reputation: A global perspective. Public Relations Review, 42(1), 79-81. https://doi.org/10.1016/j.pubrev.2015.11.004

Alloza, Á., Carreras, E., \& Carreras, A. (2013). Reputación corporativa. Lid.

Arslanagic, M., \& Zabkar, V. (2017). Hold me responsible: The role of corporate social responsibility and corporate reputation for client-perceived value. Corporate Communications: An International Journal, 22(2), 209-219.

Baraibar-Díez, E., \& Sotorrío, L. (2018). The mediating effect of transparency in the relationship between corporate social responsibility and corporate reputation. Revista Brasileira de Gestão de Negócios, 20(1), 5-21. https://doi.org/10.7819/rbgn.v20i1.3600

Bowen, H. (2013). Social responsibilities of the businessman. University of Iowa Press. https:// ebookcentral.proquest.com

Carrió, M. (2013). Gestión de la reputación corporativa: Convierte lo que piensan y dicen de ti en tu mejor activo. Libros de Cabecera. 
Carroll, A. B. (1979). A three-dimensional conceptual model of corporate social performance. Academy of Management Review, 4, 497-505.

Choongo, P. (2017). A longitudinal study of the impact of corporate social responsibility on firm performance in sMes in Zambia. Sustainability, 9(8), 1300.

CONE. (2013). Cone communications social impact study. http://www.conecomm.com/research-blog/2013-cone-communications-social-impact-study

Curras-Pérez, R. (2010). Identidad e imagen corporativas: revisión conceptual e interrelación. Teoría y Praxis, (7), 9-34.

Dahlsrud, A. (2008). How corporate social responsibility is defined: An analysis of 37 definitions. Corporate Social Responsibility and Environmental Management, 15(1), 1-13. https://doi.org/10.1002/csr.132

De Castro, M. (2008). Reputación empresarial y ventaja competitiva. EsIC.

Del Brío, J. A., \& Lizarzaburu Bolaños, E. (2018). csR actions in companies and perception of their reputation by managers: Analysis in the rural area of an emerging country in the banking sector. Sustainability, 10(4), 920. https://ideas.repec.org/a/gam/jsusta/v10y2018i4p920-d137518.html

Engizek, N., \& Yasin, B. (2017). How CSR and overall service quality lead to affective commitment: mediating role of company reputation. Social Responsibility Journal, 13(1), 111-125. https://doi.org/10.1108/SRJ-09-2015-0135

Fatma, M., Rahman, Z., \& Khan, I. (2015). Building company reputation and brand equity through CSR: the mediating role of trust. International Journal of Bank Marketing, 33(6), 840-856.

Fernández Sánchez, J., Luna Sotorrío, L., \& Baraibar Díez, E. (2015). The relationship between corporate social responsibility and corporate reputation in a turbulent environment: Spanish evidence of the Ibex35 firms. Corporate Governance, 15(4), 563-575. https://doi. org/10.1108/CG-08-2014-0101

García-Santos, J. J., \& Madero-Gómez, S. (2016). La evolución del concepto de responsabilidad social corporativa: Revisión literaria. Conciencia Tecnológica, (51), 38-46. https://www. redalyc.org/pdf/944/94446004006.pdf

González, S. (2017). Conceptualización de la reputación corporativa: nuevo enfoque y propuesta. Revista de la Asociación Española de Investigación de la Comunicación, 4(7), 130-137.

Henríquez Larrarte, R., \& Oreste Burgos, R. I. (2015). Implicancias de una responsabilidad social empresarial sustentable. Revista Electrónica Gestión de las Personas y Tecnologías, 8(23), 16-27. http://www.revistas.usach.cl/ojs/index.php/revistagpt/article/view/2224 
Hopkins, M. (2003). The planetary bargain: Corporate social responsibility matters. London: Earthscan.

Jalilvand, M., Nasrolahi, L., Kazemi, H., \& Khazaei, J. (2017). Social responsibility influence on customer trust in hotels: Mediating effects of reputation and word-of-mouth. Tourism Review, 72(1), 1-14.

Kim, H., Hur, W., \& Yeo, J. (2015). Corporate brand trust as a mediator in the relationship between consumer perception of csR, corporate hypocrisy, and corporate reputation. Sustainability, 7(4), 3683-3694.

Lee, C., Chang, W., \& Lee, H. (2017). An investigation of the effects of corporate social responsibility on corporate reputation and customer loyalty-evidence from the Taiwan non-life insurance industry. Social Responsibility Journal, 13(2), 355-369.

Lin, H., Zeng, S., Wang, L., Zou, H., \& Ma, H. (2016). How does environmental irresponsibility impair corporate reputation? A multi-method investigation. Corporate Social Responsibility and Environmental Management, 23(6), 413-423.

Molina, E., Córdova, J., Meza, E., \& López, P. (2017). La responsabilidad social empresarial en las empresas del Ecuador: un test de relación con la imagen corporativa y desempeño financiero. Ingeniería Industrial. Actualidad y Nuevas Tendencias, 5(18), 23-44.

Monitor Empresarial de la Reputación Corporativa. (2019). Ranking empresas. http://www. MERCO.info/es/ranking-MERCO-empresas

Park, J., Lee, H., \& Kim, C. (2014). Corporate social responsibilities, consumer trust and corporate reputation: South Korean consumers' perspectives. Journal of Business Research, 67(3), 295-302. https://doi.org/10.1016/j.jbusres.2013.05.016

Peñalosa Otero, M. E., \& López Celis, D. M. (2018). El estereotipo de los colombianos frente al consume socialmente responsible. Pensamiento y Gestión, (44), 243-260. http://dx.doi. org/10.14482/pege.44.10384

Perestelo-Pérez, L. (2013). Standards on how to develop and report systematic reviews in psychology and health. International Journal of Clinical and Health Psychology, 13(1), 49-57. https://doi.org/10.1016/S1697-2600(13)70007-3

Pérez, M., Espinoza, C., \& Peralta, B. (2016). La responsabilidad social empresarial y su enfoque ambiental: una visión sostenible a futuro. Revista Universidad y Sociedad, 8(3), 169-178.

Ramos, M., Manzanares, M., \& Gómez, F. (2018). The effect of technological posture and corporate social responsibility on financial performance through corporate reputation. International Journal of Innovation, 6(2), 164-179.

Ramos, S., \& Polo, M. (2018). Corporate reputation and communication management. Revista de Estudios Institucionales, 5(9), 243-252. 
Reverte, C., Gómez, E., \& Cegarra, J. (2016). The influence of corporate social responsibility practices on organizational performance: Evidence from Eco-Responsible Spanish firms. Journal of Cleaner Production, (112), 2870-2884.

Su, L., Pan, Y., \& Chen, X. (2017). Corporate social responsibility: Findings from the Chinese hospitality industry. Journal of Retailing and Consumer Services, (34), 240-247.

Taghian, M., D’Souza, C., \& Polonsky, M. (2015). A stakeholder approach to corporate social responsibility, reputation and business performance. Social Responsibility Journal, 11(2), 340-363.

Torassa, S. (2010). Más allá de la teoría: responsabilidad social empresarial en casos prácticos. Incae Business Review, 1(11), 70-78.

Valenzuela, L., Jara, M., \& Villegas, F. (2015). Prácticas de responsabilidad social, reputación corporativa y desempeño financiero. Revista de Administração de Empresas, 55(3), 329-344.

Vázquez, J. (2012). La responsabilidad social corporativa en los principales grupos de comunicación españoles: Incorporación, gestión y análisis de la información a través de sus páginas web. Correspondencias y Análisis, (2), 111-128.

Verčič, A., \& Ćorić, D. (2018). The relationship between reputation, employer branding and corporate social responsibility. Public Relations Review, 44(4), 444-452.

Vidaver, D., \& Brønn, P. (2015). Reputation, responsibility, and stakeholder support in Scandinavian firms: A comparative analysis. Journal of Business Ethics, 127(1), 49-64.

Vlastelica, T., Kostic, S., Okanovic, M., \& Milosavljevic, M. (2018). How corporate social responsibility affects corporate reputation: Evidence from an emerging market. Journal of East European Management Studies, 23(1), 10-29.

Wood, D. J. (1991). Corporate social performance revisited. Academy of Management Review (16), 691-718. 